Hydrocephalus

\section{"Normal pressure hydrocephalus": what's in a name?}

\section{Dunn}

\section{A nomenclature based on pathophysiology would improve treatment}

n their paper in this issue (pp 9-12), Bret et al $^{1}$ question the concept of normal pressure hydrocephalus (NPH) and propose renaming the condition chronic hydrocephalus. The original description of the condition is usually attributed to Hakim and Adams, ${ }^{2}$ although, as Bret and colleagues point out, the condition was probably described earlier. The original description was of a constellation of clinical symptoms and signs of symptomatic hydrocephalus without overt evidence of raised intracranial pressure rather than a defined pathophysiology.

The underlying pathology is heterogeneous in these patients, including those with so called "idiopathic NPH", and this in turn has led to many of the problems of nomenclature and difficulties in patient selection and treatment.

Bret and colleagues argue convincingly that NPH is in many ways a misnomer. Cerebrospinal fluid (CSF) pressure is not "normal" in these patients. There are well defined abnormalities of CSF dynamics. NPH is not a cause of dementia, as rigorously defined, but rather associated with specific patterns of subcortical cognitive deficit. It is not a condition confined to the elderly. There seem to be examples of similar clinical presentations in young adults and children.

From a clinical perspective there is a need to identify patients who are likely to benefit from some form of CSF diversion-traditionally ventriculoperitoneal shunting-and, equally important, to identify patients unlikely to benefit. Shunting, particularly in elderly patients, is associated with a significant incidence of both acute and cumulative longer term complications. A number of tests, including tests of CSF dynamics and a variety of imaging investigations, have been proposed to aid in predicting responsiveness to shunting. The positive predictive value of many of these tests is high but often the negative predictive value is less impressive. To date there is probably no single test that adds substantially to the predictive power of clinical features and routine computed tomography or magnetic resonance imaging.

A nomenclature based on pathophysiology may improve patient classification and selection and may guide the application and development of more helpful preoperative tests. I am not convinced that the term "chronic hydrocephalus" offers much improvement over NPH in this regard.

A greater understanding of pathophysiology may also point the way to new forms of treatment. A recent report of the use of endoscopic third ventriculostomy in three patients with "NPH"4 suggests that it would be unwise to assume that the only effective treatment is ventriculoperitoneal shunting.

It is apparent that the term "normal pressure hydrocephalus" has a number of deficiencies, but I am not sure that "chronic hydrocephalus" is that great an improvement.

J Neurol Neurosurg Psychiatry 2002;73:8

\section{Authors' affiliations}

L Dunn, Institute of Neurological Sciences, Southern General Hospital, 1345 Govan Road, Glasgow G51 4TF

Correspondence to: Mr L Dunn Itd 1x@udcf.gla.ac.uk

\section{REFERENCES}

1 Bret P, Guyotat J, Chazal J. Is normal pressure hydrocephalus a valid concept in 2002? A reappraisal in five questions and proposal for a new designation of the syndrome as "chronic hydrocephalus". J Neurol Neurosurg Psychiatry 2002:73;9-12.

2 Adams RD, Fisher CM, Hakim S, et al. Symptomatic occult hydrocephalus with "normal" cerebrospinal fluid pressure. N Engl J Med 1965;273:117-26.

3 Corkill RG, Cadoux-Hudson TA. Normal pressure hydrocephalus: developments in determining surgical prognosis. Curr Opin Neurol 1999;12:671-7.

4 Mitchell P, Mathew B. Third ventriculostomy in normal pressure hydrocephalus. $\mathrm{Br} J$ Neurosurg 1999;13:382-5.

\title{
Online index
}

A pdf (printer friendly) version of the index for volume 72 of JNNP will be posted on the journal's web site (www.jnnp.com) in conjunction with the July issue (Vol 73, Issue 1). This pdf will be freely available to all. 